Théologiques

Théologiques

\title{
Englobement et autonégation
}

\section{La portée réelle des références de Nishida à la théologie chrétienne}

\section{Jacynthe Tremblay}

Volume 20, numéro 1-2, 2012

Les philosophes de l’École de Kyōto et la théologie

URI : https://id.erudit.org/iderudit/1018855ar

DOI : https://doi.org/10.7202/1018855ar

Aller au sommaire du numéro

\section{Éditeur(s)}

Faculté de théologie et de sciences des religions, Université de Montréal

ISSN

1188-7109 (imprimé)

1492-1413 (numérique)

Découvrir la revue

Citer cet article

Tremblay, J. (2012). Englobement et autonégation : la portée réelle des références de Nishida à la théologie chrétienne. Théologiques, 20(1-2), 83-102. https://doi.org/10.7202/1018855ar

\section{Résumé de l'article}

L'intérêt de Nishida Kitarō (1870-1945) pour la théologie chrétienne répond à un but essentiellement philosophique. À travers son utilisation de thèmes théologiques, il s'efforça d'approfondir ses propres concepts philosophiques et de montrer qu'une juste compréhension du fait religieux repose sur une compréhension adéquate de sa « logique du lieu »(場所的論理, basho-teki ronri). Il parvint ainsi à établir une chose très importante pour la compréhension de l'être humain : le soi est un lieu. Non limité au statut de sujet connaissant classifiant des objets de connaissance et détaché de l'aspect intuitif de la connaissance, le soi devient le lieu même de la rencontre avec Dieu. Il peut alors se posséder dans ce qui le dépasse absolument au fondement de lui-même et s'affirmer, comme le fait Dieu lui-même, au sein de l'autonégation, devenant alors un individu véritable ou un soi véritable. 


\title{
Englobement et autonégation
}

\section{La portée réelle des références de Nishida à la théologie chrétienne}

\author{
Jacynthe Tremblay* \\ Philosophie \\ Université d'Hokkaidō (Sapporo, Japon)
}

Au vu de la très grande quantité des références de Nishida Kitarō (18701945) aux théologiens chrétiens, il est manifeste que le but de cet article ne saurait être d'analyser de manière exhaustive la pensée de chacun d'entre eux, de même que tous les thèmes théologiques utilisés par Nishida, mais plutôt d'en montrer la visée générale, dans la perspective d'ensemble de son œuvre, et de porter attention aux thèmes philosophiques présents dans les contextes où il évoque la théologie chrétienne. La raison en est que l'intérêt diffus de Nishida pour la religion, qui s'est étendu de 1891 jusqu'en 1945, participe d'un double but, essentiellement philosophique.

Le premier d'entre eux consiste dans la recherche de ce qui réside au fondement de sa propre philosophie. Cela ne signifie en aucune manière que cette dernière soit fondée sur la religion, mais plutôt que la recherche du fondement a pu pousser Nishida à utiliser des termes tirés de la religion afin de viser un domaine situé au-delà de la connaissance et qui soit susceptible de la rendre possible et de l'examiner sous toutes ses ramifications (voir ci-après la section 1). En d'autres termes, il se servit à l'occasion de notions à connotation religieuse afin de tenter de repousser l'horizon sur lequel le domaine de la connaissance ainsi que tous les autres ordres de la réalité puissent être réexaminés d'une manière nouvelle et, si possible, hors

* Jacynthe Tremblay est docteur en théologie (Université de Montréal, 1990) et chercheuse invitée à l'Université d'Hokkaidō (Sapporo, Japon). Ses recherches actuelles portent sur Nishida Kitarō et sur la philosophie japonaise contemporaine en général. Parmi ses publications, on compte (2007) Auto-éveil et temporalité. Les Défis posés par la philosophie de Nishida, Paris, L'Harmattan. 
du cadre de la relation subjectivo-objective habituelle en philosophie et dans les sciences humaines en général.

Le second but poursuivi par Nishida, à travers ses références aux théologiens chrétiens et à certains thèmes de la théologie, fut de tenter d'approfondir ses propres thèmes philosophiques et, en particulier, de montrer qu'une juste compréhension du fait religieux repose sur une compréhension adéquate de sa "logique du lieu» (場所的論理, basho-teki ronri) (voir les sections 2 à 5). Formulée de la manière la plus simple, celle-ci s'appuie sur le fait que «tout ce qui est se situe dans quelque chose» (Nishida 2008, 223). J'aurai l'occasion de revenir en détail sur ce type de logique, dans la suite de cet article, puisque la portée des références de Nishida à la théologie chrétienne ne peut être bien comprise que dans le cadre d'une explication de celle-ci. Disons simplement ici qu'elle est une manière de classer les différents niveaux de la réalité (par exemple, ici, la raison scientifique, la conscience morale et la conscience religieuse, chacune d'entre elles étant plus vaste que la précédente et l'englobant) les uns par rapport aux autres. Alors que la logique fonctionne habituellement par définition de concepts, la logique du lieu «situe» les différents faits conceptuels et réels par rapport au lieu (場所, basho) précis dans lequel ils adviennent, et en relation à ce lieu. De cette manière, il est aussi possible de comprendre les diverses interrelations entre ces faits.

Cette insistance mise par Nishida sur le lieu et corrélativement sur l'aspect «englobant» de sa philosophie dans ses références à la théologie chrétienne va bien au-delà de la simple comparaison que beaucoup de commentateurs de Nishida ont tenté d'établir entre les "sources bouddhiques» de cet auteur et le christianisme. D'ailleurs, une analyse détaillée (que je n'entreprendrai pas ici) de ses quelques références au bouddhisme permettrait de montrer que celles-ci poursuivent le même but du côté de sa tradition asiatique, à savoir l'approfondissement de sa propre perspective philosophique, caractérisée par la logique du lieu et par le caractère englobant de celle-ci.

\section{Analyse quantitative des références de Nishida à la théologie chrétienne}

Dans une première approximation, les allusions de Nishida à la théologie chrétienne ${ }^{1}$, bien qu'elles soient nombreuses, peuvent être perçues comme

1. Celles-ci comprennent également les références aux propos proprement dits du Christ (NKZ 1: 129, 160, 186; NKZ 2: 259, 349; NKZ 3: 513; NKZ 7: 428, 431, 447; NKZ 8: 62, 246; NKZ 9: 60, 145, 334; NKZ 10: 146; NKZ 11: 142, 410, 428, 
décevantes en ceci qu'elles manquent de systématisation, voire simplement d'unité. Une exception notable est son étude d'Augustin², dont la pensée lui servit d'inspiration constante pour le développement de sa conception de la temporalité (時間性, jikan-sei) (Nishida 2008; voir Tremblay 2007b; 2008). Il convient de s'y attarder brièvement puisqu'elle permettra de commencer à comprendre le caractère englobant de la logique du lieu.

Partant de la question posée par Augustin, au livre XI des Confessions, à savoir "Qu'est-ce donc que le temps? » (Augustin 1964, 264), Nishida suivit le même parcours que ce dernier pour aboutir à la notion de "maintenant éternel» (永遠の今, eien no ima). Il montra que les apories séculaires du temps mises en relief par Augustin peuvent trouver leur résolution dans une conception renouvelée de la notion de présent (現在, genzai) comme site même de la temporalité. À terme, ce présent, compris comme lieu englobant, se retrouva lui-même englobé par le stade ultime de la temporalité nishidienne, à savoir le maintenant éternel. À la différence d'Augustin, cependant, Nishida délogea le maintenant éternel de sa position au-delà de l'histoire et le réarticula en lien avec le temps historique, établissant par la même occasion qu'il est marqué au plus haut degré par le caractère englobant qui constitue le propre de la logique du lieu.

Nishida se servit également de la pensée d'Augustin (notamment des Soliloques, de La Cité de Dieu et du De Trinitate) pour développer sa notion centrale d' " autoéveil» (自覚, jikaku) (Nishida 1978b; 2003a; voir Tremblay 2007b). Il estima que c'est Augustin qui, le premier, parvint à saisir la véritable nature de l'esprit. Il s'agit ici de ce que la tradition philosophique qualifia à rebours, en référence explicite à Descartes, de «cogito d'Augustin », rebaptisé par Nishida "autoéveil d'Augustin » afin de bien marquer ses différences avec Descartes. L'admiration que Nishida portait

432, 443, 461-462; NKZ 14: 315) et de Paul (NKZ 1: 156, 169, 192, 405 ; NKZ 2 : 349; NKZ 3: 288; NKZ 6: 181, 290; NKZ 7: 427; NKZ 8: 246-247; NKZ 11: 427). Ces indications réfèrent aux pages des 19 volumes qui composent les œuvres complètes de Nishida (Nishida 1978a). Ces mentions sont aussi complètes que possible. Il est entendu que plusieurs mentions d'un même auteur peuvent apparaître dans une même page.

2. NKZ 1: 142, 165, 184, 186, 405; NKZ 2: 259, 285, 288, 297, 302, 328, 347; NKZ $3: 69,452$; NKZ 4: 29, 31-32, 35, 42, 85, 155, 232, 238, 317, 364; NKZ 5: 286, 290, 311, 405; NKZ 6: 39, 49, 51, 65, 106, 112, 116, 132, 139, 176, 181, 183, 185, 193, 195, 209, 220, 229, 234, 409, 421; NKZ 7: 11, 144, 350, 409, 432; NKZ 10: 84, 240, 505; NKZ 11: 133, 156, 345, 410; NKZ 12: 19-24, 30, 34, 39, 79, 89, 112-113, 115, 117-118, 205-206, 243; NKZ 15 : 108; NKZ 17: M. 32, 5.6; NKZ 18 : T. 14, 283. 
à Augustin sur ce sujet vient du fait que ce dernier se révéla capable de réfléchir radicalement à propos du soi (自己, jiko) lui-même et de la personnalité (人格, jinkaku), et d'avoir porté cette réflexion à un haut niveau philosophique. Autrement dit, Augustin excéda le seul aspect que retint plusieurs siècles plus tard Descartes, à savoir l'aspect conscient intellectuel du soi.

En recherchant ce qu'il appelle le «soi autoéveillé»(自覚的自己, jikaku-teki jiko), Nishida fut porté par sa lecture d'Augustin à insister également sur le «monde historique» (歴史的世界, rekishi-teki sekai). Dans sa tentative d'élucidation de la notion d'autoéveil (laquelle caractérise fondamentalement le soi véritable), il passa de la philosophie de la subjectivité ayant marqué la modernité, à une philosophie du monde historique dans lequel l'être humain s'autoéveille (自覚する, jikaku suru) à travers ses multiples relations (au monde, à autrui, aux choses, voire à Dieu) (voir la section 5). Conçu ainsi de manière historique, le soi s'élargit jusqu'à devenir un «lieu » relationnel; par rapport à ce avec quoi il est en relation, ce soi véritable (真の自己, shin no jiko) se retrouve beaucoup plus englobant que le moi de l'ego cogito intellectuel.

Nishida fit appel à d'autres théologiens médiévaux et de l'époque de la Renaissance, quoique de manière beaucoup plus implicite et occasionnelle que dans le cas d'Augustin. Il s'agit d'Albert Le Grand ${ }^{3}$, de Jacob Bœhme ${ }^{4}$, de Nicolas de Cues ${ }^{5}$, de Jean Duns Scot ${ }^{6}$, d'Eckhart ${ }^{7}$, d'Érigène ${ }^{8}$, d'Origène $^{9}$, du Pseudo-Denys ${ }^{10}$ et de Thomas d'Aquin ${ }^{11}$. Sous l'influence

3. NKZ 12: 205.

4. NKZ 1: 99, 177, 186-187, 190-191, 366; NKZ 2: 182, 274, 287, 301; NKZ 3: 393, 473; NKZ 5 : 182; NKZ 6: 55; NKZ 11: 130, 197, 323, 463; NKZ 14: 318; NKZ 15: 111; NKZ 17: M. 39, 3.1, 11. 26, S. 18, S. 19, 5.7; NKZ 18: T. 14, 283.

5. NKZ 1: 99, 190; NKZ 11: 138-139; NKZ 15: 111; NKZ 17: S. 18, 2. 26 ; NKZ 19: S. 19, 291.

6. NKZ 1: 126, 187; NKZ $4: 265 ;$ NKZ 12: 205.

7. NKZ 1: 185-186, 192, 366; NKZ 6: 159, 181-182; NKZ 14: 311-327; NKZ 15: 110; NKZ 17: M. 38, M. 40, 1. 1. Voir surtout Nishida (1978d).

8. NKZ 2: 275-276, 278-280, 283-284, 299-300, 317, 341; NKZ 3: 191; NKZ 4: 154; NKZ 7: 172, 393; NKZ 8: 426, 586; NKZ 10: 361; NKZ 15: 109.

9. NKZ 2: 285, 287, 342; NKZ 15: 108; NKZ 17: T. 11.2.1.

10. NKZ 1: 189; NKZ 2: 275, 278-279, 283, 300, 317, 341; NKZ 4: 229; NKZ 5: 412, 471; NKZ 7: 432; NKZ 14: 316; NKZ 15: 108; NKZ 17: S. 19, 5.7.

11. NKZ 1: 187, 353; NKZ 4: 265; NKZ 7: 433; NKZ 10: 461; NKZ 12: 23, 204, 205. Voir surtout Nishida 1978c. 
de ces derniers, mais aussi et de manière remarquable de Plotin ${ }^{12}$ (204269), qui exerça une très forte influence sur le mysticisme médiéval, Nishida développa, lors de la première période de sa pensée (1911-1926), un volontarisme religieux.

Par son recours au néoplatonisme chrétien, Nishida dota temporairement sa notion de soi d'un arrière-fond mystique, surtout dans son livre de 1917 intitulé Intuition et réflexion dans l'autoéveil (NKZ 2: 278-335) et dans son article "Le mysticisme» de 1914 (Nishida 1978e). Compris comme unité de la pensée (思惟, shii) et de l'intuition (直観, chokkan), le soi se situe ultimement dans un abîme d'obscurité correspondant au Ungrund ou Urgrund de Bœhme. Aveuglante comme chez le PseudoDenys, cette obscurité ouvre le soi sur une infinie profondeur qui lui octroie véritablement signification et être. Cependant, ce à quoi Nishida s'arrêta ultimement, à travers ces références, fut la recherche, dans une perspective demeurant proprement philosophique, d'un troisième élément, différent de la sensibilité et de la raison, qui puisse les contenir et les expliquer. C'est ce type de totalité (全体, zentai) que le mysticisme s'efforça d'intuitionner, une autorité ou fondement interne permettant l'établissement de la connaissance, une intuition originelle transrationnelle plutôt qu'irrationnelle.

Bien qu'évoqué parfois par Nishida en langage mystique, ce domaine lui-même n'a rien de mystique ou de mystérieux. Il s'agit en réalité du stade le plus proche, le plus évident et le plus immédiat, attesté par l'expérience de tout un chacun, tout en demeurant inaccessible à la simple connaissance conceptuelle (概念的知識, gainen-teki chishiki). En effet, il n'existe pas pour Nishida de conscience spéciale, même lorsqu'elle est religieuse: "Ce qui est mystérieux n'est d'aucune utilité pour notre vie pratique. Si la religion était la conscience spéciale d'un être humain spécial, elle ne serait rien d'autre qu'un passe-temps pour gens désœuvrés » (Nishida 2004, 259, nous traduisons).

12. NKZ 1: 290; NKZ 2: 287, 317; NKZ 3: 69, 75, 271, 273, 429-430, 518; NKZ 4 : 3, 38-40, 42-44, 71, 100, 113, 128-129, 143, 153-155, 157, 160-161, 163, 166-167, 277; NKZ 5: 158-159, 403, 410, 471; NKZ 6: 154, 208, 224, 388 ; NKZ 7: 141, 157, 431, 447; NKZ 8: 541; NKZ 9: 93, 116; NKZ 10: 262, 361, 420, 424, 506, 507; NKZ 11: 446; NKZ 12: 7, 16, 105, 108, 118 ; NKZ 14: 313, 316-318, 320; NKZ $15: 103,105,107$; NKZ 17: S. 11. 7.10, S. 11. 10.7, S. 13.4; NKZ 18: T. 11, 244 ; NKZ 19: S. 4, 656, S. 11, 474, S. 13, 21. 
Parmi les auteurs chrétiens, Nishida mentionna encore au passage les réformateurs Luther ${ }^{13}$ et Calvin ${ }^{14}$, fit référence accessoirement à François $\mathrm{d}^{\prime}$ Assise ${ }^{15}$, s'attarda plus particulièrement à Pascal ${ }^{16}$ et fit quelques mentions de théologiens qui furent ses contemporains, par exemple Karl Barth ${ }^{17}$, Rudolf Bultmann ${ }^{18}$, Friedrich Gogarten ${ }^{19}$ et Paul Tillich ${ }^{20}$. Enfin, il examina soigneusement l'expérience religieuse à travers sa lecture de William James ${ }^{21}$ et de ses deux romanciers de prédilection, à savoir Dostoïevski ${ }^{22}$ et Tolstoï ${ }^{23}$.

\section{Le «lieu» du phénomène religieux}

Il est un écrit de Nishida particulièrement important lorsqu'il s'agit de comprendre les relations entre ses évocations de termes théologiques chrétiens et le développement de sa logique du lieu. Il s'agit d'un essai écrit en 1945 , peu de temps avant sa mort, et intitulé «Logique du lieu et conception religieuse du monde" (Nishida 2004).

Dans ce texte très long ( 71 pages en japonais) et touffu, Nishida résume toute sa philosophie, incluant sa logique du lieu, sa conception de l'individu (個人, kojin), du monde historique, de l'auto-éveil, de la temporalité, de l'intuition agissante (行為的直観, kōi-teki chokkan), de la culture (分化, bunka) et de l'État (国家, kokka). Il y discute de théologie dialectique et dialogue brièvement avec Karl Barth et Paul Tillich. Les thèmes théologiques proprement dits qu'il aborde sont nombreux, à savoir par exemple la création, la faute originelle, la révélation, la rédemption, la

13. NKZ 7: 425, 427; NKZ 9: 65, 216; NKZ 11: 140, 424; NKZ 14: 317; NKZ 15: 111.

14. NKZ 10: 449.

15. NKZ 1: 402-403, 406; NKZ 3: 288, 350; NKZ 14: 314.

16. NKZ 1: 117; NKZ 3: 290, 507; NKZ 7: 138, 321, 427; NKZ 8: 157, 428, 439; NKZ 9: 270; NKZ 10: 53; NKZ 11: 426, 429, 452; NKZ 12: 24, 126-127; NKZ $14: 322$.

17. NKZ 11: 398, 427.

18. NKZ 8: 565 .

19. NKZ 6: 417, 436; NKZ 8: 102; NKZ 17: S. 10, 3.17, S. 20. Voir surtout son analyse de la relation je-tu (Nishida 2003; Tremblay 2004).

20. NKZ 11: 425, 461, 463.

21. NKZ 15: 433; NKZ 17: M. 37. 1.8; NKZ 18: M. 35, 59, M. 38, 67.

22. NKZ 1: 406, 416; NKZ 3: 304; NKZ 7: 426, 440; NKZ 11: 413, 449-451, 462; NKZ 12: 180.

23. NKZ 3: 326; NKZ 12: 180; NKZ 14: 316. 
mort, la Trinité et le Christ, mais jamais de manière approfondie. Ses quelques évocations du bouddhisme sont de même niveau.

Comme il a été évoqué dans l'introduction, il y a certainement dans cet essai matière à comparaison entre le christianisme et le bouddhisme, angle d'approche que la plupart des commentateurs ont adopté. Or, la visée de l'essai se situe ailleurs, à savoir dans la mise en rapport, comme son titre l'indique, entre la logique du lieu nishidienne et sa propre conception religieuse du monde. Autrement dit, Nishida place cette dernière dans le cadre de sa logique du lieu, ainsi qu'il l'énonce lui-même clairement à quelques reprises. Partant par exemple du «retournement» du soi ou de la conversion, il stipule que «la religion ne peut être saisie, du point de vue philosophique, qu'à l'aide de la logique du lieu » (Nishida 2004, 337, nous traduisons).

Je suivrai donc la trame de la logique du lieu, dans l'essai «Logique du lieu et conception religieuse du monde », en prenant appui pour mon interprétation sur l'ensemble de la philosophie de Nishida et sur mes écrits antérieurs la concernant. Nishida lui-même ne guide que très peu ses lecteurs dans leur traversée de son essai. C'est pourquoi je m'efforcerai de montrer de quelle manière il effectue à plusieurs niveaux le passage d'un lieu moins englobant à un lieu qui l'est davantage et de quelle manière, simultanément, le soi change de statut lorsqu'il est englobé dans un lieu plus vaste, devenant lui-même plus élargi et plus authentique, jusqu'à se faire à son tour le lieu même de la relation avec un «tu» (汝, nanji), humain ou divin.

Au début de son essai, Nishida fait allusion aux savants qui se font une gloire d'affirmer que le sentiment religieux n'existe pas. Ils ont néanmoins raison au niveau où ils se situent, qui est celui de la compréhension scientifique du monde. Du point de vue scientifique, la religion est considérée comme non scientifique et irrationnelle, si ce n'est comme une intuition mystique. Par rapport à cela, le but de Nishida fut d'opérer un déplacement et d'indiquer le «lieu» précis du phénomène religieux. Qu'est-ce à dire?

L'auteur affirme en début d'analyse qu'une religion sans Dieu n'existe pas. Il considère celui-ci comme le concept fondamental de la religion. Le contact avec Dieu suppose toutefois une faculté appropriée. De même que pour l'œil, la couleur apparaît en tant que couleur et que pour l'oreille, le son apparaît en tant que son, "Dieu se manifeste à notre soi en tant que fait au niveau de l'esprit. Il n'est pas envisageable au seul niveau intellectuel. Ce qui n'est pensé qu'intellectuellement n'est pas Dieu» (Nishida 
2004, 295, nous traduisons). Ainsi, Nishida «situe » d'entrée de jeu et très précisément le phénomène de la religion comme un fait advenant «au fond» du cœur (心, kokoro) et se situant «au niveau» de l'esprit (心霊, shinrei). Cet accent sur le site de la religion est beaucoup plus important pour Nishida que la construction de la religion à partir de systèmes philosophiques $^{24}$.

Voilà clairement établi le «lieu» de la religion. Lorsque l'on parle de «lieu », cependant, on songe immédiatement à un lieu spatial. En réalité, plusieurs des lieux évoqués par Nishida sont spatiaux: le monde matériel (物質的世界, busshitsu-teki sekai), le monde biologique (生物的世界, seibutsu-teki sekai) et le monde historique (歴史的世界, rekishi-teki sekai) en font partie. En ce qui concerne ce dernier, son caractère spatial est moins clairement fixé, car il s'agit d'un lieu où des faits se produisent. En ce sens, l'agir et la liberté ont besoin d'un lieu d'exercice qui n'est plus entièrement spatial, voire qui ne l'est plus du tout.

Ce qu'il importe ici de retenir est qu'aucun des lieux susmentionnés ne peut être entièrement compris à partir de son propre niveau. C'est la raison pour laquelle Nishida pose un lieu plus vaste et plus englobant que tous, à savoir le «lieu du néant absolu» (絶対無の場所, zettai-mu no basho). Bref, lorsqu'on passe d'un lieu donné à un lieu plus englobant qui contient le premier, le caractère spatial du lieu devient de plus en plus ténu. On se retrouve alors devant le paradoxe présenté par un lieu dont les limites deviennent de plus en plus vastes et indéterminables, fait que Nishida cherchait à exprimer en répétant que le néant absolu (絶対無, zettai-mu) est tel un cercle sans circonférence, dont le centre est partout et la circonférence, nulle part. Dans ces conditions, il ne saurait être une chose objectivable, quantifiable, catégorisable.

Comme Nishida le définira dans la troisième section de l'essai «Logique du lieu et conception religieuse du monde» (Nishida 2004, 327337), le véritable lieu du sentiment religieux deviendra celui du néant

24. De l'avis de Nishida, même Kant — qui, dans les Fondements de la métaphysique des mœurs, est parvenu à bien élucider la conscience morale du point de vue philosophique et à bien saisir l'essence de la conscience esthétique — n'a pas réussi à voir ce qui se trouve au-delà du beau formel et a situé la religion uniquement sur le plan de la conscience morale, sans être capable aller jusqu'à la conscience religieuse. Nishida en conclut que "la religion n'a pas sa place dans la blosse Vernunft» (Nishida 2004, 296). Il n'est donc possible de parler du problème religieux ni au niveau de la conscience scientifique ni à celui de la logique objective, puisqu'il ne s'y pose même pas. 
absolu, ce qui signifie qu'ultimement, l'esprit en tant que lieu précis du phénomène religieux se situera à son tour dans ce lieu beaucoup plus vaste, englobant et indéterminable au moyen de la connaissance, scientifique ou autre. Cela signera pour lui la fin de tout repère, qu'il soit conceptuel, symbolique, ou tout simplement spatial.

Il est important ici de préciser ceci: que le lieu du néant absolu soit simultanément celui de l'expérience religieuse ne signifie aucunement que le néant absolu soit l'équivalent de ce que les religions appellent «Dieu». Nishida lui-même ne fit jamais cette identification de nature objectivante. Il lui arriva d'écrire explicitement que "Dieu est le néant absolu». Cependant, si on se fie à la structure de la langue japonaise et à la conception du monde que cette dernière implique, cette formulation n'indique pas une identification au niveau des essences, mais le fait que Dieu, tout être suprême soit-il, est marqué par le néant. Autrement dit, il ne peut être délimité par aucun prédicat. On verra aussi dans la section 4 que néant lui-même, Dieu est marqué par une autonégation (自己否定, jiko-hitei) qui le porte à se vider de lui-même pour aller vers l'humain, Nishida situant cette caractéristique au même niveau que la kénose chrétienne.

\section{Le soi face à la négation absolue}

Avant d'approfondir le problème de la religion en tant que tel et de présenter un Dieu marqué par la négation absolue (絶対否定, zettai-hitei), Nishida s'attarda dans la deuxième partie de l'essai «Logique du lieu et conception religieuse du monde» (Nishida 2004, 311-327) à la question du soi. Selon lui, le sentiment religieux se produit dans le cadre des relations processuelles entre le fini et l'infini ou, encore, entre le relatif et l'absolu (絶対者, zettai-sha). Il surgit lorsque l'existence même du soi est remise en question. Ici, le lien avec la logique du lieu est que le sentiment religieux est reconduit à son propre site d'advenance, c'est-à-dire la vie du soi lui-même.

Nishida insiste encore une fois sur le fait que ce sentiment religieux n'est pas de l'ordre d'un processus de décision procédant par une suite de déductions conceptuelles rigoureuses. "Toutes les erreurs et défauts de compréhension de la religion, dit-il, viennent de ce qu'on la pense du point de vue de la logique abstraite (抽象的論理, chūshō-teki ronri)» (Nishida 2004, 363, nous traduisons). Pour quelle raison en va-t-il ainsi ? Parce que le niveau de l'existence est beaucoup plus vaste et englobant que le domaine rationnel. C'est la raison pour laquelle Nishida y situe la vie du soi. 
Lorsqu'il insiste sur le fait que le sentiment religieux se situe au niveau de l'esprit, il ne vise pas le soi conscient (意識的自己, ishiki-teki jiko) ou rationnel, mais un type de soi plus élargi — il parle encore du véritable soi individuel — qui se trouve au niveau de l'existence elle-même plutôt qu'à celui des oppositions métaphysiques que sont le fini et l'infini, le relatif et l'absolu.

La métaphysique et, avec elle, la théologie ont habituellement procédé par hiérarchisation des différents ordres de la réalité qu'elles ont situés en opposition irréconciliable. À la position supérieure correspondait toujours une position inférieure. Au plus s'opposait toujours le moins: au bien le mal, à la transcendance l'immanence ou, encore, à l'esprit la matière. La logique du lieu de Nishida opère de manière très différente. Les oppositions traitées en métaphysique de manière antagoniste sont placées par ce philosophe au niveau d'un continuum où loin d'être incommunicables, elles se conditionnent l'une l'autre. Alors que la représentation métaphysique de la réalité était bidimensionnelle, avec en haut la série "supérieure » des oppositions habituelles et en bas la série «inférieure» (comme par exemple dans l'opposition esprit-matière), la philosophie de Nishida pourrait être qualifiée de tridimensionnelle, au sens où elle est fondée sur une série de lieux, tous plus englobants les uns par rapport aux autres.

Nishida fait un pas supplémentaire en affirmant que l'existence du soi devient problématique lorsque celui-ci s'autoéveille à l'autocontradiction qui lui est inhérente et qui est précisément la raison d'être du soi. Le problème de la religion (comme celui de la philosophie d'ailleurs) surgit lorsque nous nous rendons compte de la réalité de cette autocontradiction. Partant, et c'est là le fil qui conduira sous peu à la très importante notion d'autonégation, le fait fondamental de l'existence du soi consiste dans l' «autoéveil de la mort» (c'est-à-dire dans l'évidence absolue et incontournable de la mort), dont la connaissance seule permet à l'être humain de savoir qu'il est un véritable individu. Ici, l'argumentation de Nishida est très claire. S'il situe le problème de la religion et celui du sentiment religieux au niveau de l'existence, et s'il décèle précisément dans cette dernière une autocontradiction dont la mort est l'avatar suprême, c'est parce qu'il s'agit précisément là de l'un des niveaux les plus englobants qui constituent l'être humain.

Il a été mentionné que le sentiment religieux ne peut se produire au niveau de la raison, puisqu'elle est un lieu trop restreint pour ce type d'expérience, quoiqu'elle ait une importance déterminante au niveau qui est le sien. Pour marquer la différence entre le niveau de la raison et celui 
de l'existence, Nishida mentionne explicitement ce qui suit: "Mais originairement, le rationnel et l'universel ne sont pas des choses vivantes. Et il n'y a pas de mort pour ce qui ne peut pas vivre. La raison ne s'autoéveille pas à la mort» (Nishida 2004, 313, nous traduisons). Cela lui permet d'aboutir au propos central de l'essai «Logique du lieu et conception religieuse du monde", à savoir que le soi s'autoéveille à sa mort éternelle seulement lorsqu'il fait face à l'absolu, c'est-à-dire à une «négation absolue » prenant la forme d'une autonégation.

Ici, la connexion avec la logique du lieu est plus difficile à percevoir. Cette dernière est quand même très présente si l'on considère que la notion de négation absolue est la version, au niveau de cette logique, de la mort absolue. Dans la logique du lieu, en effet, la seule manière de passer d'un lieu plus restreint (par exemple celui de la raison) à un lieu plus englobant est l'autonégation. J'ai abondamment traité de cette notion dans d'autres écrits (Tremblay 2007a; 2007b). On prendra la mesure de son importance en mentionnant simplement ici que Nishida dit en avoir fait le point de départ de toute sa philosophie. À la différence de la mort absolue, elle n'advient pas uniquement au terme de la vie terrestre; elle est le moteur même de la mise en relation de termes contradictoires ou simplement opposés (comme le «je» et le «tu»). Il ne s'agit certainement pas de la part du soi d'une autoannihilation, mais plutôt d'une manière de laisser derrière soi ses acquis pour s'ouvrir à des expériences d'un autre type. Autrement dit, le soi rationnel, celui qui se positionne à titre de sujet devant des objets, doit se nier pour être en mesure de se transformer en l'un ou l'autre des soi plus englobants établis par Nishida, à savoir le soi volontaire (意志的自己, ishi-teki jiko), le soi agissant (行為的自己, kōi-teki jiko) ou le soi religieux (宗教的自己, shūkyō-teki jiko). En se niant lui-même, le soi ne fait pas que s'élargir. Il devient simultanément un «lieu » plus englobant lui permettant de contenir en lui des «contenus» (内容, naiyō) plus vastes que de simples objets de connaissance, à savoir par exemple, le sentiment religieux et l'autoéveil à la mort éternelle ou absolue par laquelle il est marqué. L'autonégation est aussi la seule manière pour l'être humain d'entrer en relation avec Dieu puisque se niant lui-même, il s'ouvre à la rencontre avec lui.

Cela dit en reprenant les termes de Nishida: «Ce n'est pas par la simple réflexion que s'autoéveille notre soi [...] il se connaît au sein de l'autonégation" (Nishida 2004, 314, nous traduisons), ou encore, il «se possède dans l'autonégation" (Nishida 2004, 329, nous traduisons). En effet, la vérité du soi se trouve dans la connaissance de son néant éternel. 
Son existence se trouve là où il vit en étant marqué par la mort. En ce sens, l'autonégation et la mort deviennent le lieu même de l'existence du soi.

\section{L'absolu qui se possède dans l'autonégation}

Jusqu'à maintenant, Nishida a traité du sentiment religieux du point de vue de l'existence de l'être humain, lequel est marqué par la mort et par l'autonégation. Or, il en va de même de Dieu. Nishida parvient à éclaircir ce point en reprenant la perspective précédente, rappelant que l'humain peut rencontrer Dieu à travers la mort. Celle-ci advient lorsque le relatif s'oppose à l'absolu, devenant ainsi lui-même néant. Comme l'exprime Nishida, et l'affirmation est ici d'importance: "C'est uniquement par la mort que notre soi touche à Dieu et se rattache à lui sous le mode de la correspondance inverse» (Nishida 2004, 315, nous traduisons).

L'expression 《correspondance inverse» (逆対応, gyaku-taiō) se retrouve surtout dans l'essai "Logique du lieu et conception religieuse du monde». Elle est la reprise, réservée à la relation entre Dieu et l'être humain, d'une autre notion très importante dans la philosophie de Nishida, celle d' "auto-identité absolument contradictoire»(絶対矛盾的自己同一, zettai-mujun-teki jiko-dōitsu). Celle-ci cherche à rendre compte de la mise en relation des termes contradictoires plutôt qu'à insister sur leur caractère prétendument irréconciliable. À preuve de son statut dans la philosophie de Nishida, celui-ci va jusqu'à affirmer que «le monde religieux est concevable uniquement en tant qu'autodétermination du lieu qu'est l'autoidentité absolument contradictoire, uniquement au moyen de la logique du lieu " (Nishida 2004, 329, nous traduisons).

Combinée à la logique du lieu, la logique de l'auto-identité absolument contradictoire répond au fait qu'aucune identité (同一, doiitsu) n'est fixée une fois pour toutes. Elle est mue par une nécessité interne, celle de l'autonégation, à se déconstruire incessamment afin de se rendre apte à la rencontre avec l'autre (他者, tasha). À travers celle-ci, l'auto-identité de l'être qui s'est soumis à l'autonégation se trouve reconstruite d'une manière sans cesse nouvelle. Cela ne signifie aucunement que le soi serait tout simplement nié jusqu'à perdre sa propre identité. D'un point de vue religieux, cela ne signifie pas non plus qu'il deviendrait Dieu ou le Bouddha, par rapprochement ou par identification.

Dans le cadre des relations entre l'absolu et l'être humain, la notion d'auto-identité absolument contradictoire de même que sa version religieuse de «correspondance inverse» signifient que l'absolu, loin de se 
situer par-delà toute opposition, comporte avec le soi une racine identique. Nishida remet dans le même mouvement en question la transcendance absolue (絶対的超越, zettai-teki chōetsu) de Dieu soutenue par Barth, si tant est que l'absolu doive, à l'instar de l'être humain, contenir en lui une autonégation absolue. Cette conception nishidienne se retrouve condensée dans des formules percutantes:

L'absolu se possède nécessairement dans l'autonégation [...] Dieu se trouve nécessairement en ce monde sous le mode de l'autonégation. En ce sens, il est absolument immanent. On peut donc affirmer de lui qu'il ne se trouve nulle part en ce monde, mais que simultanément, il n'existe aucun endroit où il ne se trouve pas. (Nishida 2004, 316, nous traduisons)

Comme l'humain, Dieu est marqué par le néant. Pour devenir humain, il doit se nier lui-même, de la même manière que le contact avec Dieu requiert de la part de l'humain une autonégation atteignant son point culminant avec la mort.

Il n'en demeure pas moins qu'étendue à Dieu la logique de l'autoidentité absolument contradictoire de même que la notion d'autonégation, qui en est le moteur, risquent de heurter de front la notion théologique d'immutabilité divine. Elle pourrait impliquer que Dieu est imparfait, car soumis à une série de changements consécutifs à des rencontres incessantes avec l'autre qu'est l'être humain. La perspective de Nishida, rappelons-le, est toutefois tributaire d'un autre type de logique que celle, aristotélicienne et thomiste, qui fonde la théologie traditionnelle. Elle cherche à rendre compte des relations entre l'humain et Dieu autrement qu'on ne l'avait fait jusque-là.

À ce point de son argumentation, Nishida introduit la notion de kénose. En effet, le Dieu véritable ne pouvant être autosuffisant dans sa transcendance, il doit être absolument kénotique. Autrement dit, le Dieu absolument transcendant doit être simultanément absolument immanent. Par autonégation, il se démet de soi-même jusqu'à devenir absolument autre, c'est-à-dire humain et de ce fait, immanent à ce monde. Il n'est donc pas un Dieu transcendant et arbitraire, mais un Dieu créateur qui, grâce à l'autonégation dont il est marqué, est toujours déjà apte au face à face avec sa création.

Nishida insiste sur le fait qu'il ne s'agit pas là de panthéisme, chose dont on pourrait l'accuser si on essayait de penser Dieu suivant une logique objective, mais plutôt d'un exemple de la manière dont il réussit à mettre en relation les termes contradictoires, en l'occurrence Dieu et l'humain, 
lesquels ne risquent plus désormais d'être cantonnés dans une opposition irréconciliable ni d'être confondus de manière panthéiste.

Un autre exemple frappant de la manière particulière selon laquelle Nishida gère les termes contradictoires est lorsqu'il assène (d'une manière passablement surprenante, vue du côté de la théologie chrétienne) ceci : du fait qu'il renferme en lui une autonégation absolue, «le Dieu véritablement absolu doit être sous son autre aspect démoniaque» (Nishida 2004, 321, nous traduisons). La raison en est qu'un Dieu qui ne ferait que s'opposer au mal et lutter avec lui ne serait qu'un Dieu relatif, alors que le Dieu véritable, du fait qu'il contient en lui la négation absolue, doit pouvoir s'abaisser jusqu'au mal extrême. Il est un Dieu absolu dans la mesure où il sauve les impies: "L'agapè absolu doit s'étendre jusqu'au méchant absolu. Dieu se cache dans le cœur de l'humain le plus méchant aussi, sous forme de correspondance inverse " (Nishida 2004, 321, nous traduisons). Bref, Dieu n'est pas pour Nishida une chose telle que la Gottheit eckhartienne, mais plutôt une auto-identité absolument contradictoire contenant en elle-même une autonégation absolue.

Dans ce contexte, le rôle de l'autonégation est évident. Dieu est certes sans rapport avec le mal lorsqu'on cherche à lui préserver sa Gottheit et sa transcendance absolue; mais simultanément, il demeure très élevé audessus de la mêlée humaine et de ses misères. Dans ces conditions, on voit difficilement comment l'un et l'autre pourraient entretenir des relations quelconques, même et surtout sotériologiques.

En soutenant que Dieu comporte un aspect démoniaque, Nishida ne prétend pas que le démoniaque l'affecte dans son essence, ne serait-ce qu'en partie, car répétons-le, son mode de pensée se situe d'emblée au-delà d'une métaphysique des essences. Il s'agit plutôt de marquer le rapport à l'altérité qui caractérise tout être, dont Dieu, du moment qu'il comporte en lui l'autonégation. Plus encore, le mouvement d'autonégation de même que le mode relationnel qu'il permet d'instaurer occasionnent une détermination réciproque (相互限定, sōgo-gentei) de la part des éléments en relation. En d'autres termes, l'amour absolu de Dieu, condensé par Nishida dans le mot agapè, transforme l'être humain qui, devant le Dieu qui s'est nié pour venir vers lui (on parle dans la doctrine chrétienne de révélation ou autocommunication de Dieu, de même que de kénose), rejettera progressivement le mal.

Dans ces conditions, l'humain, point de projection de Dieu, est luimême auto-identité contradictoire entre le bien et le mal. S'inspirant ici comme ailleurs de Dostoïevski, Nishida présente le cœur humain comme 
un champ de bataille, celui des deux duellistes que sont le diable et Dieu (Nishida 2004, 322 ; voir Dostoïevski 1962, 132). Or, c'est précisément là, sur ce champ de bataille, que se trouve l'existence du soi comme être volontaire et comme personnalité. Du côté du divin, il est plus malaisé de parler d'une détermination (限定, gentei) ou d'une transformation de l' "auto-identité » divine qui serait consécutive à, ou adviendrait à travers, ses relations à l'être humain. Et pourtant, c'est déjà le cas, du fait même que dans l'incarnation, Dieu est devenu humain.

Dans ce qui précède, il est frappant de constater l'absence de disparités entre l'humain et Dieu. Tout autant que Dieu, l'humain est grevé par une autonégation absolue. Comme Dieu, le mode fondamental de son existence est celui de la relation (関係, kankei). De la même manière que Dieu, au moyen d'une autonégation rendue absolue par l'absolu de son amour, s'est abaissé vers l'humain, ce dernier, se niant à son tour, afin de se rendre apte à entrer en relation avec Dieu, peut — suivant le mouvement de la logique $\mathrm{du}$ lieu — s'agrandir graduellement aux dimensions de ce que Bœhme a appelé l' "abîme ", et qui sous-tend et soutient son existence, sans pour autant en arriver jamais à coïncider totalement avec lui. Il y a auto-identité de Dieu et auto-identité de l'humain, mais il s'agit d'une auto-identité absolument contradictoire, parce que marquée par une autonégation permettant tant à Dieu qu'à l'humain d'entrer en relation avec l'autre, l'un et l'autre voyant alors sa propre identité reconstruite toujours à nouveau dans et à travers sa relation à l'autre.

\section{Le lieu où se trouve le soi}

Jusqu'à maintenant, Nishida s'est appliqué à élucider la nature du sentiment religieux. Celui-ci ne consiste pas pour l'être humain à essayer d'atteindre par ses propres forces un Dieu inaccessible et isolé dans sa transcendance absolue, mais à prendre sérieusement en compte l'autonégation qui le fonde, à réfléchir profondément au sujet de son propre principe ou de son propre fond.

On considère souvent que l'expérience religieuse est un but à atteindre et que le soi est égaré tant qu'une telle chose n'est pas réalisée. Or, dit Nishida, en ancrant une fois de plus le problème de la religion dans sa logique du lieu, «l'égarement ne consiste pas pour le soi à s'égarer de son but, mais à s'égarer du lieu où il se trouve» (Nishida 2004, 323, nous traduisons). Il dit encore de manière plus explicite: "Qu'est-ce que notre soi ? Où se situe-t-il ? Tel est le problème essentiel du soi lui-même, le pro- 
blème du lieu où il se trouve " (Nishida 2004, 327, nous traduisons). Ainsi, le véritable lieu de la religion n'est pas un paradis (chrétien ou bouddhique) inaccessible, mais la profondeur du soi lui-même.

On objectera peut-être que cette conception nishidienne de la religion est passablement anthropocentriste. Sa juste compréhension dépend toutefois de la conception de l'humain qui est ici en jeu et, plus encore, du type de logique par le biais de laquelle on étudie l'humain religieux. Les propos précédents ont insisté ponctuellement sur cette question. Ni l'humain ni Dieu ne sont des "objets" classifiables et déterminables suivant une logique objective, dès lors qu'il s'agit d'examiner des faits qui se trouvent au niveau de l'existence même du soi. L'intention de Nishida était de remettre en question les substantialisations indues (notamment celle du soi) lorsqu'il est question du rapport à Dieu :

Les diverses méprises à propos des relations entre Dieu et l'humain proviennent elles aussi de la manière de voir de la logique objective. Je ne suis pas quelqu'un qui rejette la logique objective [...] Mais l'erreur se trouve là où on considère comme des substances ce que pense la logique abstraite; elle se trouve dans la «substantialisation des concepts». (Nishida 2004, 329 330 , nous traduisons)

Que le problème de la religion ait son origine dans le lieu même où se trouve le soi et du fait d'une descente de ce dernier dans sa propre profondeur abyssale ne signifie pas que la religion se réduirait au soi lui-même, au sens où il serait une substance ou un fondement (Nishida 2004, 329) tentant de comprendre de manière conceptuelle des objets de connaissance tels que «religion", «Dieu », «faute» ou «rédemption». Le soi véritable dont parle Nishida est avant tout relationnel, mode auquel il accède, précisément, par le biais de l'autonégation. Plutôt qu'un point de référence autocentré, il devient le «lieu » du sentiment religieux et se situe à son tour lui-même dans son propre «lieu» qu'est le monde historique où il naît, où il vit et où il meurt. Nishida en arrive ainsi à montrer, à travers sa recherche $\mathrm{du}$ «lieu» où se trouve le soi, que l'essence de ce dernier consiste à se nier soi-même et donc à exister hors de soi dans le monde historique. De ce fait, il possède le pouvoir d'exprimer absolument en lui-même le monde, tout en s'autodéterminant de manière créatrice comme l'un de ses foyers.

Il est possible de vérifier, par ce genre de propos, que le but de Nishida dans son recours à la théologie est non pas de parler de Dieu lui-même, mais de cerner davantage le propre de l'être humain et sa place dans le monde. Sa pensée, répétons-le, n'en est pas pour autant anthropocentriste 
puisque dans sa recherche du lieu où se trouve le soi, il cherche à montrer qu'il est avant tout en relation et surtout, qu'il n'exerce ni primauté ni maîtrise sur tous les types de relations (au monde, aux choses, au «tu », à Dieu) qu'il peut entretenir.

Une fois le soi délogé de la place prédominante qu'il a occupée durant la modernité et qui consistait pour lui à voir le monde à partir de soimême, il apparaît que c'est le monde historique qui est le fait premier. Nishida passe par le fait même à un soi agissant et volontaire se situant "dans» le monde, à un soi faisant partie, avec d'autres soi, d'un monde historique commun où toute rencontre est rendue possible, dont la rencontre avec Dieu: "Nous sommes des réalités religieuses dans la mesure où nous sommes des réalités historiques qui naissons dans le monde historique, agissons dans le monde historique et mourons dans le monde historique» (Nishida 2004, 354, nous traduisons). Formule redondante s'il en est, mais qui est le reflet de l'insistance marquée de Nishida sur le fait que la rencontre avec le divin ne s'effectue pas dans un ailleurs inaccessible, qu'il soit supramondain ou situé au terme de l'histoire, mais dans le «lieu » même où l'humain naît, vit et meurt, à savoir le monde historique.

Même la tentative nishidienne de fonder une théologie sur sa logique du lieu a un caractère historique: "Une théologie qui relève de la logique du lieu n'est ni théiste ni déiste. Ni fait de l'esprit ni fait de la nature, elle est historique» (Nishida 2004, 322, nous traduisons). Situé au niveau du monde historique, le soi est beaucoup plus large que son avatar limité au niveau de la connaissance. Le soi comme sujet connaissant (知識的自己, chishiki-teki jiko) peut certes connaître nombre de choses sur Dieu ou discourir abondamment à son sujet. Cependant, c'est lorsqu'il s'élargit suffisamment, lorsqu'il adopte un mode relationnel en se situant dans le monde historique, qu'il se rend apte à tout type de rencontre, dont la rencontre avec Dieu.

Mentionnons en terminant un dernier thème sur lequel Nishida luimême insiste souvent dans l'ensemble de son œuvre, puisqu'il représente le point culminant de la logique du lieu. Il s'agit de l'amour (愛, ai), auquel il parvient à travers l'importante question de l'approfondissement du soi. Car, en définitive, tout le détour par le néant absolu, l'autonégation et la recherche du fond abyssal du soi avait pour fin d'y retracer le sentiment religieux dissimulé «au fond du cœur de tout humain », bien que beaucoup ne s'en rendent pas compte. D’où la nécessité de la conversion — engagée à partir de l'extrémité d'une volonté affinée - qui permet à l'humain égaré de revenir en son lieu propre, c'est-à-dire au fond de lui-même, de retour- 
ner à sa propre origine, c'est-à-dire l'absolu, là où se trouve un appel de Dieu. Pour appuyer son propos, Nishida fait ici appel à Augustin, qui affirme au début des Confessions: "Vous nous avez créés pour vous [et] notre cœur est inquiet jusqu'à ce qu'il repose en vous» (Augustin 1964, 15; voir NKZ 11:410; NKZ 12: 20). À cet appel, l'être humain répond à travers sa propre décision. "Adhérer à la foi, en conclut Nishida, consiste donc pour l'humain à obéir à la décision de Dieu à travers sa propre décision» (Nishida 2004, 342, nous traduisons).

De telles relations religieuses s'établissent entre, d'une part, ce qui dépasse absolument le soi et le fait néanmoins s'établir et, d'autre part, un soi absolument unique, individuel et volontaire. À ce niveau, la logique du lieu opère encore et de manière éminente puisque l'absolu, compris désormais comme le véritable lieu du soi, englobe (包む, tsutsumu) absolument ce dernier, même lorsqu'il tente de se dérober. Nishida va encore plus loin en affirmant finalement ici la primauté de l'amour, dont la forme véritable consiste dans une relation d'auto-identité absolument contradictoire entre un « je » et un «tu»:

J'affirme ici également que notre soi s'oppose à l'absolu comme individu unique et dans son aspect volontaire. Car l'amour aussi doit être une relation d'auto-identité contradictoire entre deux personnalités opposées. L'amour absolu consiste à englober absolument ce qui est contraire à soi. Le soi volontaire, qui est une existence absolument autocontradictoire, se trouve confronté - sous le mode de l'auto-identité contradictoire - à la racine de son établissement à ce qui le fait s'établir. Là, notre soi doit entrer en contact avec l'amour absolu qui l'englobe. Le soi personnel ne s'établit pas à partir d'une simple opposition d'ordre volontaire. Pour cette raison, Dieu est en un certain sens amour, dans quelque religion que ce soit (Nishida 2004, 344-345, nous traduisons).

\section{Conclusion}

Voilà à quoi il est possible de parvenir, en suivant la trame de la logique du lieu, dans l'essai «Logique du lieu et conception religieuse du monde ». La question qui préoccupait Nishida, à savoir d' "où " provient le sentiment religieux et «par quoi » il est fondé, s'en trouve résolue d'une manière différente que si on en faisait l'un des problèmes cognitifs de la connaissance objective ou l'un des problèmes moraux concernant les devoirs du soi (voir Nishida 2004, 327). Ainsi, le but premier de Nishida, à travers ses évocations de la théologie chrétienne, dans le cadre de sa logique du 
lieu, et qui consistait à cerner ce qui se trouve à la base de sa pensée philosophique, est désormais atteint avec la mise en évidence d'un soi élargi aux dimensions du néant absolu ou, encore, du soi véritable.

Il découle de toute l'argumentation nishidienne une chose très importante pour la compréhension de l'être humain, affirmée comme un leitmotiv de 1926 à 1945 : le soi est un lieu (Nishida 2004, 352). Non limité au statut de sujet connaissant (認識主観, ninshiki-shukan) classifiant des objets de connaissance (認識対象, ninshiki-taishō) et détaché de l'aspect intuitif de la connaissance, non réduit lui-même au statut d'objet pouvant ultimement être connu, le soi devient le lieu même de la connaissance objective, tout autant que de la rencontre de l'autre. Il peut alors se posséder dans ce qui le dépasse absolument au fondement de lui-même et s'affirmer au sein de l'autonégation. S'approfondissant aux dimensions du néant absolu — ou s'établissant à partir de la négation absolue, comme le veut la logique du lieu — il devient un individu véritable ou un soi véritable.

\section{Références}

Augustin (1964), Les Confessions, Paris, Garnier-Flammarion (GF 21).

Dostoї́nski, F. (1962), Les Frères Karamazov, t. 1, Paris, Hachette.

NishidA, K. (1978a), 『西田幾多郎全集』(Euvres complètes de Nishida Kitarō) (NKZ) (19 volumes), Tōkyō, Iwanami Shoten.

(1978b)，「アウグスチヌスの自覚」(L'autoréveil d'Augustin), dans NKZ 12, p. 112-118.

(1978c),「トマスアクイナスの全集」 (Les ceuvres complètes de Thomas d'Aquin), dans NKZ 12, p. 204-206.

(1978d),「エックハルト神秘説と一灯園生活」(Le mysticisme d'Eckhart et l'Ittō-en), dans NKZ 14, p. 311-327.

(1978e),「神秘主義」(Le mysticisme), dans NKZ 15, p. 102-113.

(2003), "Je et tu», dans K. NishidA, L’Éveil à soi / trad. par J. Tremblay, Paris, CNRS Éditions, p. 95-144.

- (2004), 「場所的論理と宗教的世界観」 ( Logique du lieu et conception religieuse du monde»), dans 『西田幾多郎全集 (Euvres complètes de Nishida Kitarō) 〈第 10 巻 volume 10〉哲学論文集第 六・哲学論文集第七・「続思索と体験」以後』 (essais philosophiques, 6 - essais philosophiques, 7 - pensée et expérience, suite), Tōkyō, Iwanami Shoten, p. 295-366. 
(2008), «L'autodétermination du maintenant éternel » / trad. par J. Tremblay, dans J. Tremblay, dir., Laval Théologique et Philosophique. Philosophie japonaise du XX $X^{e}$ siècle, 64, p. 245-276.

Tremblay, J. (2004), «La relation "je-tu” dans la philosophie de Nishida ", Religiologiques, 29, p. 117-151.

(2007a), autoéveil et temporalité. Les Défis posés par la philosophie de Nishida, Paris, L'Harmattan.

(2007b), L'Être-soi et l'être ensemble. L'autoéveil comme méthode philosophique chez Nishida, Paris, L'Harmattan.

\section{Résumé}

L'intérêt de Nishida Kitarō (1870-1945) pour la théologie chrétienne répond à un but essentiellement philosophique. À travers son utilisation de thèmes théologiques, il s'efforça d'approfondir ses propres concepts philosophiques et de montrer qu'une juste compréhension du fait religieux repose sur une compréhension adéquate de sa 《logique du lieu»(場所的論理, basho-teki ronri). Il parvint ainsi à établir une chose très importante pour la compréhension de l'être humain: le soi est un lieu. Non limité au statut de sujet connaissant classifiant des objets de connaissance et détaché de l'aspect intuitif de la connaissance, le soi devient le lieu même de la rencontre avec Dieu. Il peut alors se posséder dans ce qui le dépasse absolument au fondement de lui-même et s'affirmer, comme le fait Dieu lui-même, au sein de l'autonégation, devenant alors un individu véritable ou un soi véritable.

\section{Abstract}

Nishida Kitarō's interest (1870-1945) for Christian theology is above all philosophical. Through his use of theological topics, he sought to deepen his own philosophical concepts and to show that a proper understanding of religion is based on a proper understanding of his «logic of place» (場所的 論理, basho-teki ronri). He thus managed to establish something very important for the understanding of the human being: the self is a place. Not limited to the status of a knowing subject classifying objects and separated from the intuitive aspects of knowledge, the self becomes the place of the encounter with God. Then, it is able to possess itself in what transcends itself, and to assert itself, as God made himself, in the self-negation, thus becoming a true self or a true individual. 